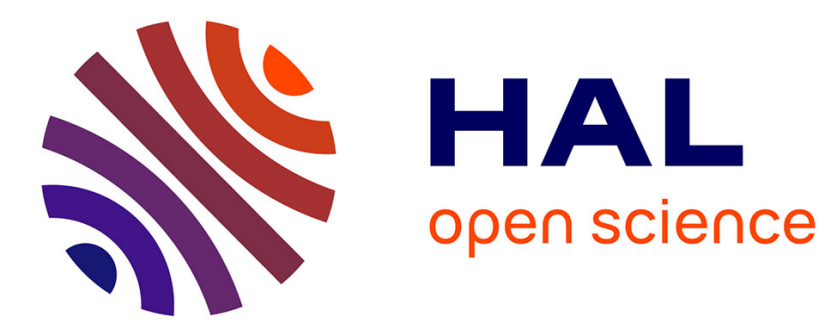

\title{
A Delay-Based Sustained Chemical Oscillator: Qualitative Analysis of Oregonator-Based Models
}

Hakki Ulas Unal, Islam Boussaada, Silviu-Iulian Niculescu

\section{To cite this version:}

Hakki Ulas Unal, Islam Boussaada, Silviu-Iulian Niculescu. A Delay-Based Sustained Chemical Oscillator: Qualitative Analysis of Oregonator-Based Models. IEEE Life Sciences Letters, 2017, 3 (3), pp.9 - 12. 10.1109/LLS.2017.2756834 . hal-01656725

\section{HAL Id: hal-01656725 \\ https://hal-centralesupelec.archives-ouvertes.fr/hal-01656725}

Submitted on 17 Dec 2018

HAL is a multi-disciplinary open access archive for the deposit and dissemination of scientific research documents, whether they are published or not. The documents may come from teaching and research institutions in France or abroad, or from public or private research centers.
L'archive ouverte pluridisciplinaire HAL, est destinée au dépôt et à la diffusion de documents scientifiques de niveau recherche, publiés ou non, émanant des établissements d'enseignement et de recherche français ou étrangers, des laboratoires publics ou privés. 


\title{
A Delay Based Sustained Chemical Oscillator: Qualitative Analysis of Oregonator Based Models
}

\author{
Hakki Ulaş Ünal and Islam Boussaada and Silviu-Iulian Niculescu Senior Member, IEEE
}

\begin{abstract}
Belousov-Zhabotinskii reaction is a complicated chemical oscillator exhibits temporal and spatiotemporal behaviours that quantitatively match some rhythmic behaviours in biological systems. Its important features are covered by a simple irreversible mechanism, called the Oregonator. By taking into account the physical constraints and to describe the mechanism with a fewer variables, two-delays Oregonator based model is considered in order to investigate the delay effects in chemical reactions exhibiting sustained oscillations. It is shown that the solutions of the model, obtained by use of the delay-mass action kinetics, are positive and bounded. By utilizing the asymptotic stability properties of the model which exhibits positive and bounded solutions, a positive invariant set for the solutions is described. Numerical results are presented to compare with the existing solutions which are qualitatively matches with the experimental results.
\end{abstract}

Index Terms-Chemical Oscillator, Oregonator, Delayed Negative Feedback, Sustained oscillation

\section{INTRODUCTION}

The recurrence of any event within a biological system at some intervals can be considered as a biological rhythm [1]. It can be observed from in a single cell to physiological process with periods ranging from fractions of a second to years. Although rhythmic phenomena has been known for a long time, their mechanism has not been fully understood. Observation of analogous behaviours in some chemical oscillators leads to explain the biological rhythm by investigating the mechanism of these oscillators [2]. However, these reactions involve dozens of species and intermediate steps and it may not be possible to fully cover the features of their dynamics without resorting to modelling and numerical computations [3].

Mass-action kinetics are used to model most of the biological and chemical reactions. The law of mass-action states that the velocity of a reaction is proportional to product of the concentrations of reactants raised to the power of their stoichiometric coefficients [4]. This leads to obtain a

H. U. Ünal is with Laboratoire des Signaux et Systemes, CentraleSupélec, 3 rue Joliot Curie, 91192, Gif-sur-Yvette, France, on leave from the Dept. of Electrical and Electronics Eng., Anadolu University, Turkey e-mail: (e-mail: huunal@anadolu.edu.tr; unalha@12s.centralesupelec.fr). The work of H. U. Ünal was partially supported by Scientific Research Projects Commission of Anadolu University under grant $1505 \mathrm{~F} 238$.

I. Boussaada is with Institut Polytechnique des Sciences Avancées, 7 rue Maurice Grandcoing, 94200 Ivry-sur-Seine, France (e-mail: islam.boussaada@12s.centralesupelec.fr).

S.-I. Niculescu is with Laboratoire des Signaux et Systemes, CNRSCentraleSupélec-Université Paris Sud, 3 rue Joliot-Curie, 91192 Gif-surYvette, France, (e-mail: silviu.niculescu@12s.centralesupelec.fr)

The authors would like to thank Marc R. Roussel from Department of Chemistry and Biochemistry, University of Lethbridge, Canada. model expressed by polynomial ordinary differential equations (ODEs) with positive solutions for positive initial conditions [5]. As suggested in [6], delayed mass-actions kinetics lead to more accurate models by conserving the simplicity and a relative reduced number of parameters (see [7]-[9] and references therein). In addition, models described by using delay-differential equations (DDEs) exhibit better consistency compared to ODEs from the numerical point of view [10], [11]. In fact, delays naturally appear in dynamics of chemical reactions due to the transportation and/or propagation phenomena. They are often relatively small and may be neglected for analysis, since solution of a DDE requires to define an infinite-dimensional set of initial functions on a time interval of length equal to the largest delay. However, since the presence of delay in systems' dynamics often induce unexpected behaviours [12], they should be taken into account. On the other hand, they may not be necessarily used for certain reversible chemical reactions.

Belousov-Zhabotinskii (BZ) reaction is one of the complicated chemical oscillators exhibits sustained and relaxation oscillatory behaviours that are analogous to those in biological systems. Its irreversible mechanism is described through a simple model, called Oregonator [13]. To the best knowledge of the authors', delay effects in Oregonator based models have not been deeply analyzed in the literature. In this work, we will make a qualitative and quantitative analysis for a two delay Oregonator based model given in [14]. In Section II, the mechanism of the model be presented. Then, in Section III, positivity and boundedness properties of the solutions of the model will be presented. Its asymptotic stability properties and a positive invariant set for the solutions of the model will be given in the same section. Numerical results will be presented in Section IV and the paper will be concluded in Section V.

\section{Delay MODEl of The OREgonator}

The BZ reaction can be described as presence of two different processes occurring at the same time. One of these processes occurs when the bromide ion concentration rises above certain value, while the other one dominates the reaction when the concentration falls below a certain value. The oscillatory behaviour origins that whenever bromide ion concentration is sufficiently enough, consumes the bromide ion that leads to initiate the other process which indirectly yields to produce bromide ion. This mechanism can be described by the Oregonator model, whose kinetic equations are as following 
[13]:

$$
\begin{aligned}
& \mathrm{A}+\mathrm{Y} \stackrel{k_{1}}{\longrightarrow} \mathrm{X}+\mathrm{P} \\
& \mathrm{X}+\mathrm{Y} \stackrel{k_{2}}{\longrightarrow} 2 \mathrm{P} \\
& \mathrm{B}+\mathrm{X} \stackrel{k_{3}}{\longrightarrow} 2 \mathrm{X}+\mathrm{Z} \\
& 2 \mathrm{X} \stackrel{k_{4}}{\longrightarrow} \mathrm{A}+\mathrm{P} \\
& \mathrm{Z} \stackrel{k_{5}}{\longrightarrow} f \mathrm{Y}
\end{aligned}
$$

where $A, B$ are assumed to be constant chemical species, $Y$ (Bromide ion), $X$ (Bromous acid), and $Z$ (Cerium (IV)) are chemical variables, $P$ is some chemical product, $k_{i}, i=$ $1, \ldots, 5$, is the positive rate constant, and, $f$ is a stoichiometric factor. By the law of mass-action, the mechanism can be described by a set of ODEs with these chemical variables.

In [15], a positive delayed feedback Oregonator, which is based on one of the models in [16], has been analyzed. The models in [16] cover the relaxation oscillatory mechanism of the Oregonator by two variables, which correspond to "slow" and one of the "fast" dynamics of the mechanism. By use of delay mass-action kinetics, a few attempts have been done to describe the sustained oscillatory behaviour of the Oregonator with two variables in [6], [17]. However, in our opinion, the proposed models in these works are not realistic, in addition, one of the models in [17] does not exhibit oscillatory behaviour, while, the other one, is not meaningful in the sense of law of mass-action.

The reactions start whenever the reactants posses some amount of energy, called "activation energy", which naturally happens after a some amount of time. In addition to this, the crucial argument is that molecules, which are activated and have the required minimum activation energy, have not to react immediately [18]. Then, in our opinion, it will be more realistic to consider the outputs of $\left(R_{1}\right)$ appear after a some amount of delay that implies $Y$ in this step will be subjected to a delay, what we call "delayed concentration". This term is completely different than the used in [17] from the modelling and physical point of view. In [14], in addition to this delay, another delay, which arises to represent the Oregonator with two variables as in [6], also taken into account. By the use of delay-mass-action kinetics, dynamics of the model in [14] can be expressed as;

$$
\begin{aligned}
& \dot{x}(t)=\eta\left(y\left(t-\tau_{1}\right)-x(t) y(t)+x(t)-q x(t)^{2}\right) \\
& \dot{y}(t)=\alpha\left(-y(t)-x(t) y(t)+f x\left(t-\tau_{2}\right)\right)
\end{aligned}
$$

where $\tau_{1}$ is introduced due to the physical constraints and $y\left(t-\tau_{1}\right)$ is called "delayed-concentration" of bromide-ion, $\tau_{2}$ is introduced to ensure the sustained oscillatory behaviour, $x=$ $X k_{2} / \alpha, y=Y k_{2} / \eta, \alpha=k_{1} A, \eta=k_{3} A, q=2 k_{4} \alpha /\left(k_{2} \eta\right)$.

Throughout the paper, $\mathcal{R}_{+}^{n}$ is the set of $n$-tuples with positive real entries, if $n=1$, it is represented as $\mathcal{R}_{+}$. $\mathcal{C}:=\mathcal{C}\left([-\tau, 0), \mathcal{R}_{+}\right)$, where $\tau:=\max \left\{\tau_{1}, \tau_{2}\right\}$, represents the Banach space of continuous functions mapping from $[-\tau, 0)$ into $\mathcal{R}_{+}, \mathcal{R}(\cdot)$ is the real part and $/(\cdot)$ is the argument of a complex number $(\cdot)$, and $\mathcal{C}_{+}$is the closed-right-half-plane.

\section{Qualitative/Quantitative AnAlysis}

\section{A. Properties of the dynamic model}

The unique positive equilibrium point of the dynamic model, say $\left(x^{*}, y^{*}\right)$, is $y^{*}=f^{*} x^{*} /\left(1+x^{*}\right)$, where $x^{*}$ is the unique positive solution of

$$
q^{*}\left(1+x^{*}\right) x^{*}=1+x^{*}-f^{*}\left(x^{*}-1\right),
$$

for a given $\left(f^{*}, q^{*}\right)$ iff $f^{*} \in \mathcal{R}_{+}$and $q^{*} \neq 1$.

Proposition 1: The solutions of the model with positive initial history functions are positive and bounded, if $(\eta, \alpha) \in \mathcal{R}_{+}^{2}$.

Proof: Let us assume that the positivity statement does not hold. Then, since the initial conditions and history functions are positive, at least one of the solutions crosses the zero at a finite time. Let $x(\theta), y(\theta) \in \mathcal{C}$ be initial history functions for $x(t)$ and $y(t)$ and $t_{1}$ be the first time when $x\left(t_{1}\right)$ or $y\left(t_{1}\right)$ (or both) becomes negative. Then, there exists $T \in\left(0, t_{1}\right)$ such that $x(T) y(T)=0$. Let $x(T)=0$. Then, from (1), since $\dot{x}(t)_{\mid t=T}=\eta y\left(T-\tau_{1}\right)>0$, where $y(\theta)>0, \quad \theta \in\left[-\tau_{1}, 0\right)$, $x\left(t_{1}\right)$ can not be negative. Now, let us assume that $y(T)=0$. However, from (2), since

$\dot{y}(t)_{\left.\right|_{t=T}}=\alpha f x\left(T-t_{2}\right)>0$, where $x(\theta)>0, \quad \theta \in\left[-\tau_{2}, 0\right)$,

the solutions are positive. Now, let us assume that the positive solutions are unbounded. Therefore, there exists a sequence $\left\{t_{m}\right\} \rightarrow \infty$ as $m \rightarrow \infty$ such that

$$
0<\frac{d}{d t} x(t)_{\mid t=t_{m}} .
$$

Then, since $x\left(t_{m}\right)<x\left(t_{m+1}\right)$, there exists some $M^{\prime}$ such that $x\left(t_{m}\right)>1 / q$ for $m \geq M^{\prime}$. Then, by (1) and from (4), since

$$
\begin{aligned}
y\left(t_{m}-\tau_{1}\right)+x\left(t_{m}\right) & >y\left(t_{m}\right) x\left(t_{m}\right)+q x\left(t_{m}\right)^{2} \\
& >y\left(t_{m}\right) x\left(t_{m}\right)+x\left(t_{m}\right), m \geq M^{\prime},
\end{aligned}
$$

which implies $y\left(t_{m}-\tau_{1}\right)>y\left(t_{m}\right) x\left(t_{m}\right)$.Then, (4) holds only if $\frac{d}{d t} y(t)_{\mid t=t_{p}}<0$ for some time sequence $\left\{t_{p}\right\} \rightarrow \infty$ as $p \rightarrow \infty$. However, since the initial history functions are positive and bounded, (4) does not hold, hence, $x(t)$ and $y(t)$ are bounded.

Remark 1: The equilibrium points of the model are the same as in [13], since delay terms does not have an affect on the solution of $\dot{x}(t)=\dot{y}(t)=0$. However, if we relax the positivity of $\eta$ and $\alpha$, there exists positive initial conditions that result in negative solutions.

\section{B. Stability and spectral properties}

Let us consider the model for $\left(f^{*}, q^{*}, \eta\right) \in \mathcal{R}_{+}^{3}$, where $q^{*}<$ $1, \eta^{*}>1$. Then, the characteristic function of the linearized model around $\left(x^{*}, y^{*}\right)$ by assuming $\eta \alpha=1$ can be written as

$$
\Delta\left(s, \tau_{1}, \tau_{2}\right)=P(s)-f^{*} Q_{1}\left(s, \tau_{1}\right) Q_{2}\left(s, \tau_{2}\right),
$$

where $P(s)=\left(s+\eta\left(q^{*} x^{*}+\frac{f^{*}}{1+x^{*}}\right)\left(s+\frac{1+x^{*}}{\eta}\right), Q_{1}\left(s, \tau_{1}\right)=\right.$ $\left(x^{*}-e^{-s \tau_{1}}\right)$, and $Q_{2}\left(s, \tau_{2}\right)=\left(\frac{x^{*}}{1+x^{*}}-e^{-s \tau_{2}}\right)$, where $\tau_{2} \geq 0$.

Proposition 2: $\Delta\left(s, \tau_{1}, \tau_{2}\right) \neq 0$ for $\mathcal{R}(s) \geq 0$, if any one of the following conditions hold;

i) $f^{*} \leq 1 / 2$ or $f^{*} \geq 3 / 2$, 
ii) $\tau_{2}=0$,

iii) $\tau_{1}=0$, and, $\tau_{2}<\frac{1}{f^{*}}\left(\frac{1}{\eta}+\eta q^{*}\right)$.

Proof: From (6), since $P(s)$ and $Q_{1}\left(s, \tau_{1}\right) Q_{2}\left(s, \tau_{2}\right)$ do not have common zeros for $s \in \mathcal{C}_{+}, \Delta\left(s^{*}, \tau_{1}^{*}, \tau_{2}^{*}\right)=0$ for some $\tau_{1}^{*} \geq 0, \tau_{2}^{*} \geq 0, s^{*} \in \mathcal{C}_{+}$iff

$$
\begin{aligned}
& \left|P\left(s^{*}\right)\right|=f\left|Q_{1}\left(s^{*}, \tau_{1}^{*}\right)\right|\left|Q_{2}\left(s^{*}, \tau_{2}^{*}\right)\right| \\
& \angle P\left(s^{*}\right)=\angle Q_{1}\left(s^{*}, \tau_{1}^{*}\right) / Q_{2}\left(s^{*}, \tau_{2}^{*}\right) .
\end{aligned}
$$

Then, since $x^{*}>1$, (7) does not hold for $s \in \mathcal{C}_{+}$if $f^{*} \leq 1 / 2$ or $f^{*} \geq 3 / 2$. In addition, for $\tau_{2}^{*}=0$, or $\tau_{1}^{*}=0$, but, $\tau_{2}^{*}<$ $\frac{1}{f^{*}}\left(\frac{1}{\eta}+\eta q^{*}\right)$, then, (8) fails for $s \in \mathcal{C}_{+}$.

Proposition 3: $\Delta\left(s, \tau_{1}, \tau_{2}\right)$ has a real positive zero in $\left(\gamma / \tau^{*}, 1 / \tau^{*}\right)$ for $\tau_{1} \geq \tau^{*} / \gamma, \tau_{2} \geq \tau^{*}$, where

$$
\tau^{*}>\frac{\eta}{1-e^{-1}}+\frac{\left(x^{*}\right)^{2}-1}{\eta p\left(1-e^{-1}\right)},
$$

$\gamma<1$, however, $\gamma>\log (\varphi)$, where

$$
\varphi:=\left(\frac{f^{*}\left(x^{* 2}-1\right)}{2 f^{*}\left(x^{* 2}-x^{*}-1\right)-\left(x^{*}+1\right)\left(x^{*}+2\right)}\right),
$$

where $f \in(0.8,1.1)$ and $q<<1$.

Proof: Since $\Delta\left(0, \tau_{1}, \tau_{2}\right)>0, \Delta\left(s, \tau_{1}, \tau_{2}\right)=0$ for some $s=\sigma>0$ iff there exists $\left(\sigma^{*}, \tau_{1}^{*}, \tau_{2}^{*}\right) \in \mathcal{R}_{+}^{3}$ such that $\Delta\left(\sigma^{*}, \tau_{1}^{*}, \tau_{2}^{*}\right)<0$, which implies

$$
\begin{aligned}
& \gamma^{2}+\left(\frac{\eta p^{*}}{x^{*}+1}+\frac{x^{*}+1}{\eta}\right) \tau_{2}^{*} \gamma \\
& <\tau_{2}^{* 2}\left(f^{*}\left(x^{*}-1\right)\left(\frac{x^{*}}{1+x^{*}}-e^{-\gamma}\right)-p\right) \\
& +\tau_{2}^{* 2}\left(f^{*}\left(1-e^{-\gamma \tau_{1}^{*} / \tau_{2}^{*}}\right)\left(\frac{x^{*}}{1+x^{*}}-e^{-\gamma}\right)\right),
\end{aligned}
$$

where $\gamma=\sigma^{*} / \tau_{2}^{*}$. For $\gamma \geq \log (\varphi)$, since the right-side of (11) is positive, in addition, since $0<\log (\varphi)<1$, there exists $\sigma^{*}$ such that $\sigma^{*} \tau_{2}<1$. Then, for $\tau_{1}^{*}=\tau_{2}^{*} / \gamma$ and $\tau_{2}^{*}=\tau^{*}$, which defined as in (9), the inequality holds.

Proposition 4: There exists finite intervals $\mathcal{T}$ and $\mathcal{W}$ such that $\Delta\left(j \omega, \tau_{1}, \tau_{2}\right)=0$ for $\tau_{2} \in \mathcal{T}_{2}$ and $\omega \in \mathcal{W}$ if $\tau_{1} \neq 0$. Otherwise, $\mathcal{T}_{2}$ and $\mathcal{W}$ are only single points.

Proof: Since (7) and (8) are necessary and sufficient conditions for the existence of $\left(\omega^{*}, \tau_{2}^{*}\right) \in \mathcal{R}_{+}^{2}$ such that $\Delta\left(j \omega^{*}, 0, \tau_{2}^{*}\right)=0$, and $\frac{d}{d \omega}|P(j \omega)|>0, \mathcal{T}_{2}$ and $\mathcal{W}$ contain only a single point whenever $\tau_{1}=0$. Note, for any $\left(\omega, \tau_{1}\right) \in \mathcal{R}_{+}^{2}$, since $x^{*}>1$,

$$
(x-1) \leq\left|x-e^{-j \omega \tau_{1}}\right|, \quad \angle\left(x^{*}-e^{-j \omega \tau_{1}}\right) \geq \angle\left(x^{*}-1\right) .
$$

In addition, since $\frac{d}{d \omega}\left|Q_{2}\left(j \omega, \tau_{2}\right)\right|$ (or $\frac{d}{d \tau_{2}}\left|Q_{2}\left(j \omega, \tau_{2}\right)\right|$ ) is positive, there exists some $\hat{\omega}>\omega^{*}$ such that $\Delta\left(j \hat{\omega}, \tau_{1}, \tau_{2}\right)=0$ for some $\tau_{2}^{u}>\tau_{2}>\tau_{2}^{*}$, where $\tau_{2}^{u}$ is the lower bound on $\tau_{2}$, which results in zeros in the $\mathcal{C}_{+}$. In addition, by (12), as $\tau_{1} \rightarrow \infty$, there exists some $\tilde{\tau}_{2}$ such that $\Delta\left(j \tilde{\omega}, \tilde{\tau}_{1}, \tilde{\tau}_{2}\right)=0$, where $\tilde{\tau}_{2}<\tau_{2}^{*}$.

\section{Invariance properties}

Proposition 5: Let us consider the model for which the parameters are assumed to be in the following set

$$
P:=\left\{\left(\tau_{1}, \tau_{2}, \eta, \alpha, q\right) \in \mathcal{R}_{+}^{5} \mid \tau_{2}<\tau_{1}, \frac{1}{\eta}<\alpha<1, q<1\right\} .
$$

Then, the solutions with the positive constant history functions, which are same as the initial conditions stay in

$$
\mathcal{S}:=\left\{(x(t), y(t)) \mid y(t) \leq f x(t) \leq \frac{I(0)-y(0) e^{-\eta \tau_{1}}}{q\left(1-e^{-\eta \tau_{2}}\right) / f}\right\},
$$

where $I(0)=x(0)+y(0)$.

Proof: Let $\tau_{1}=M \tau_{2}$, for some positive integer $M$. Then, by use of the method of steps,

$$
\begin{aligned}
x\left(k \tau_{2}\right)= & e^{k \tau_{2} \eta}\left(\left(I(0)-y(0) e^{-\eta \tau_{1}}\right)\right) \\
& \left.-\sum_{l=1}^{M} \int_{(l-1) \tau_{2}}^{l \tau_{2}} e^{-\eta \mu}\left(q x^{2}(\mu)+y(\mu)\right) d \mu\right) \\
& +\eta e^{k \tau_{2} \eta} \int_{(k-M-1) \tau_{2}}^{(k-M) \tau_{2}} e^{-\eta \mu} y(\mu) d \mu,
\end{aligned}
$$

which is bounded and positive by Proposition 1 . For $k \leq M$, $x\left(k \tau_{2}\right)$ strictly decreases, and, $\left(q x(t)^{2}+x(t) y(t)\right) e^{-\eta \tau_{2}}<$ $I(0)-y(0) e^{-\eta \tau_{1}}$, for $t_{1}>t \geq 0$. Note, by the arguments used in the proof of Proposition $1, y\left(k \tau_{2}\right)$ starts to decrease and $x\left(k \tau_{2}\right)$ increases whenever $k>M$. However, by the positivity of the solutions, $x\left(k \tau_{2}\right)$ can not exceed $\frac{I(0)-y(0) e^{-\eta \tau_{1}}}{q\left(1-e^{-\eta \tau_{2}}\right)}$. In addition, from (2), since $y\left(k \tau_{2}\right) \leq \frac{f x\left((k-1) \tau_{2}\right)}{1+x\left(k \tau_{2}\right)}$, the solutions, which are positive, stay in $\mathcal{S}$ if the initial conditions are in $\mathcal{S}$.

\section{Numerical Results}

By using the same rate constant parameters as in [13], the numerical solutions, which correspond to concentration of the species in the reaction, of the considered model and the model in [13] are presented in Figures 1a and 1b. As seen in these figures, the solutions are quantitatively match, where the solutions of [13] are qualitatively same as the experimentally observed oscillations in the reaction. In addition, for a chosen parameters to be in $P$, a stable limit cycle behaviour appears and solutions stay in $\mathcal{S}$ (see Fig. 1c). However, if $\tau_{1}$ is set to 0 , the solutions are asymptotically stable as seen in Fig. 1d. By using DDE-BIFTOOL [19], the stability crossing curves are obtained as presented in Fig. 1e in delay parameter space. The curve separates the delay-parameter space, where the points on the curve correspond to presence of characteristic function zeros on the imaginary axis, and the shaded region gives for which $\left(\tau_{1}, \tau_{2}\right)$ values the characteristic function has no zeros in the $\mathcal{C}_{+}$. Furthermore, the corresponding delay values for a chosen point on the curve results in sustained oscillation with a frequency, whose magnitude is sufficiently close to the chosen point [14]. For delay values above the curve presented in Fig. 1f, the asymptotic model has real zeros in the $\mathcal{C}_{+}$such that at least one of these zeros, is strictly less than $1 / \tau_{2}$ for a chosen $\left(\tau_{1}, \tau_{2}\right)$ above the shaded region in the figure.

\section{CONCLUSION}

In this work, we considered two delay-based chemical oscillator based on the Oregonator model. The solutions of the model positive and bounded for positive initial conditions and it exhibits sustained oscillations for a certain parameters. The delay, which stems from the physical constraints does not 


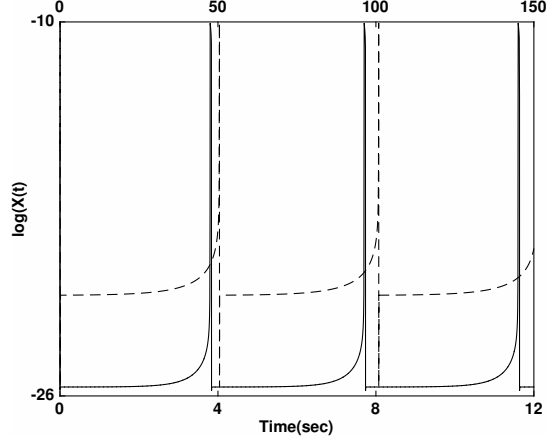

(a) Concentration of $X(t)$

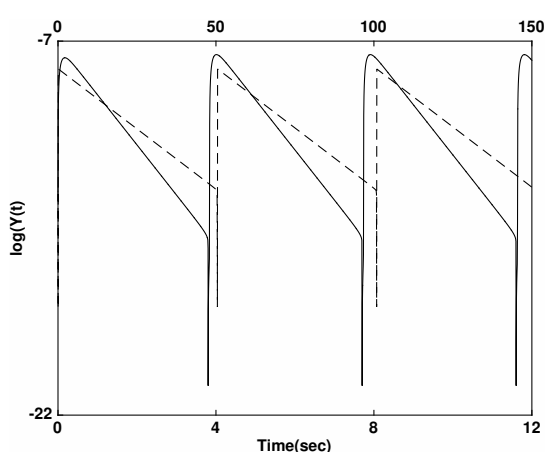

(b) Concentration of $Y(t)$

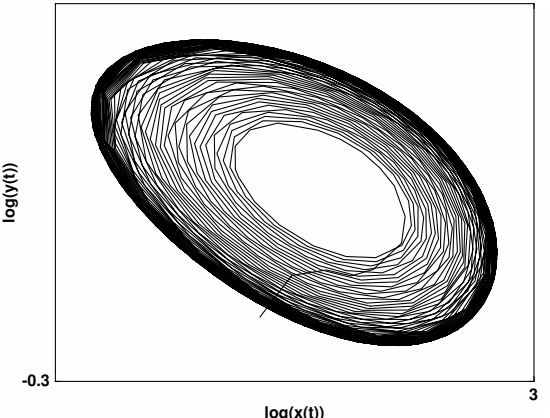

(c) Stable limit cycle if $\tau_{1} \neq 0$

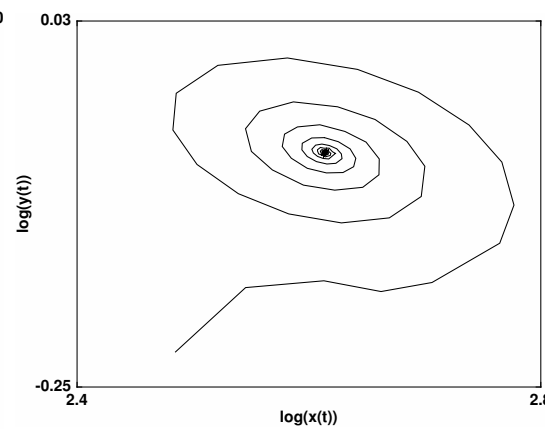

(d) Asymptotically stable solution if $\tau_{1}=0$ (f) Real zero in the $\mathcal{C}_{+}$for $\tau_{1}, \tau_{2}$ above the curve

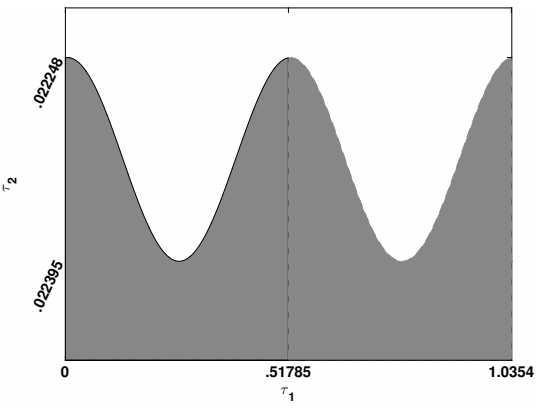

(e) Stability crossing curve

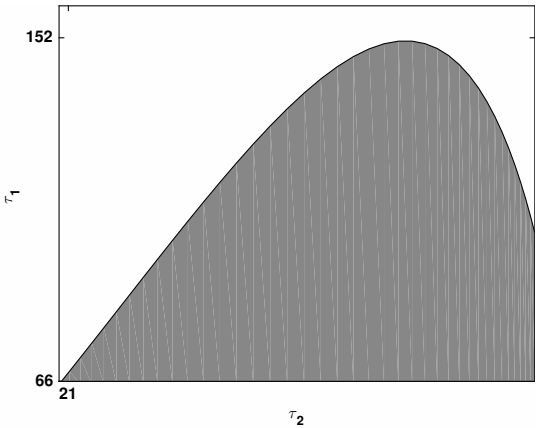

Fig. 1. In a) and b) Dashed line (-) represents numerical solution for $A=0.45 M, \tau_{1}=0.932$ sec, and $\tau_{2}=2.2501$ msec, while the straight line represents the numerical solution of the model in [13] by substituting $k_{1} A Y(t)$ in with $k_{1} A Y\left(t-\tau_{1}\right)$, where $A=0.06 M, \tau_{1}=0.932$ sec. The other parameters are taken same as in [13]. In c) Phase plane portrait of the solutions for $\alpha=0.1, \eta=1.25, f=1, q=1 / 200$, and $\tau_{1}=4.5, \tau_{2}$. In d) The parameters are same as in c) however, $\tau_{1}=0$. In e) The stability crossing curve is presented while $\tau_{1}$ versus $\tau_{2}$ for the parameters in [13]. In f) The positive real zero curve on the delay parameter space while $f$ varies in $(0.9,1,2)$ and $\eta=10, \alpha=1 / 10, q=1 / 200$.

yield instability unless the delay arising in the feedback mechanism of the model is lower some certain value. Furthermore, presence of this delay results in oscillatory behaviour with different frequencies while the delay in the negative feedback mechanism varies above a certain level.

\section{REFERENCES}

[1] J. Aschoff, "A survey on biological rhythms," in Biological Rhythms. Springer, 1981, pp. 3-10.

[2] A. Goldbeter, Biochemical oscillations and cellular rhythms: the molecular bases of periodic and chaotic behaviour. Cambridge University Press, 1997.

[3] _ , "Computational approaches to cellular rhythms," Nature, vol. 420, no. 6912, pp. 238-245, 2002.

[4] M. Feinberg, "Lectures on chemical reaction networks," https://crnt.osu.edu/LecturesOnReactionNetworks, 1979.

[5] V. Chellaboina, S. P. Bhat, W. M. Haddad, and D. S. Bernstein "Modeling and analysis of mass-action kinetics," Control Systems, IEEE, vol. 29, no. 4, pp. 60-78, 2009.

[6] M. R. Roussel, "The use of delay differential equations in chemical kinetics," The Journal of Physical Chemistry, vol. 100, no. 20, pp. 83238330, 1996.

[7] I. R. Epstein, "Delay effects and differential delay equations in chemical kinetics," International Reviews in Physical Chemistry, vol. 11, no. 1, pp. 135-160, 1992.

[8] C. J. Roussel and M. R. Roussel, "Delay-differential equations and the model equivalence problem in chemical kinetics," Phys. Can, vol. 57, pp. 114-120, 2001.

[9] M. Mincheva and M. R. Roussel, "Graph-theoretic methods for the analysis of chemical and biochemical networks. i. multistability and oscillations in ordinary differential equation models," Journal of mathematical biology, vol. 55, no. 1, p. 61, 2007.
[10] C. T. H. Baker, C. A. H. Paul, and D. R. Willé, "Issues in the numerical solution of evolutionary delay differential equations," Advances in Computational Mathematics, vol. 3, no. 3, pp. 171-196, 2013. [Online]. Available: http://dx.doi.org/10.1007/BF03028370

[11] G. A. Bocharov and F. A. Rihan, "Numerical modelling in biosciences using delay differential equations," Journal of Computational and Applied Mathematics, vol. 125, no. 1-2, pp. 183 - 199, 2000, numerical Analysis 2000. Vol. VI: Ordinary Differential Equations and Integral Equations. [Online]. Available: http://www.sciencedirect.com/science/article/pii/S0377042700004684

[12] S. I. Niculescu, Delay Effects on Stability: A Robust Control Approach, Lecture Notes in Control and Information Sciences, No. 269. London: Springer-Verlag, 2001.

[13] R. J. Field and R. M. Noyes, "Oscillations in chemical systems. IV. limit cycle behavior in a model of a real chemical reaction," The Journal of Chemical Physics, vol. 60, no. 5, pp. 1877-1884, 1974.

[14] H. U. Ünal, I. Boussaada, and S.-I. Niculescu, "Further remarks on delay dynamics in oregonator models." Kathmandu, Nepal: 12th IEEE International Conference on Control \& Automation, June 2016.

[15] K. Sriram and S. Bernard, "Complex dynamics in the oregonator model with linear delayed feedback," Chaos: An Interdisciplinary Journal of Nonlinear Science, vol. 18, no. 2, p. 023126, 2008.

[16] J. J. Tyson, "Analytic representation of oscillations, excitability, and traveling waves in a realistic model of the belousov-zhabotinskii reaction," The Journal of Chemical Physics, vol. 66, no. 3, pp. 905-915, 1977.

[17] I. R. Epstein and Y. Luo, "Differential delay equations in chemical kinetics. nonlinear models: The cross-shaped phase diagram and the oregonator," The Journal of chemical physics, vol. 95, no. 1, pp. 244 254, 1991.

[18] M. R. Wright, Fundamental chemical kinetics: an explanatory introduction to the concepts. Elsevier, 1999.

[19] K. Engelborghs, T. Luzyanina, and D. Roose, "Numerical bifurcation analysis of delay differential equations using dde-biftool," ACM Transactions on Mathematical Software (TOMS), vol. 28, no. 1, pp. 1-21, 2002. 